\title{
El Periodismo de Datos en España
}

\author{
Eva María FERreras RodríGuez \\ Universidad del País Vasco (UPV/EHU) \\ eva.ferreras@gmail.com
}

Recibido: 8 de noviembre de 2014

Aceptado: 21 de julio de 2015

\begin{abstract}
Resumen
El Periodismo de Datos se ha convertido en una de las tendencias que se están implantando en los medios. En pocos años el desarrollo y visibilidad de esta modalidad ha aumentado considerablemente y son numerosos los medios que cuentan con equipos y espacios específicos de Periodismo de Datos en el panorama internacional. Del mismo modo, existen aplicaciones, plataformas, webs, o fundaciones al margen de las empresas periodísticas cuya labor también puede ser enmarcada en este ámbito. El objetivo principal de esta contribución es establecer una radiografía de la implantación del Periodismo de Datos en España; tanto dentro como fuera de los medios. Aunque se trata de una disciplina todavía en fase de desarrollo, parece adecuado realizar un estudio exploratorio que ofrezca una panorámica de su situación actual en España.
\end{abstract}

Palabras clave: Periodismo de Datos, España, Medios de Comunicación.

\section{Data Journalism in Spain}

\begin{abstract}
The Data Journalism has become one of the trends that are being implemented in the media. In a few years the development and visibility of this modality has grown considerably and there are many media that own specific teams and spaces for Data Journalism at the international area. Similarly, there are applications, platforms, websites, or foundations outside news organizations who their work can be also framed in this area. The main target of this contribution is to establish a snapshot of the implementation of Data Journalism in Spain; inside and outside the media. Although it is a discipline still in development, it seems appropriate to perform an exploratory study that provides an overview of the current situation in Spain.
\end{abstract}

Keywords: Data Journalism, Spain, Media.

\section{Referencia normalizada}

FERRERAS RODRÍGUEZ, Eva María (2016): “El periodismo de Datos en España”. Estudios sobre el Mensaje Periodístico. Vol. 22, Núm. 1 (enero-junio), págs.: 255-272. Madrid, Ediciones Complutense.

Sumario: 1. Introducción y estado de la cuestión. 2. Material y método. 3. Periodismo de Datos: nueva modalidad informativa. 4. Análisis y resultados; 4.1. Identificación de la muestra; 4.2. Implantación del Periodismo de Datos; 4.2.1. Análisis de los medios de comunicación; 4.2.2. Análisis de las organizaciones; 4.2.3. Percepción de los profesionales. 5. Conclusiones. 6. Referencias bibliográficas.

\section{Introducción y estado de la cuestión}

Las instituciones más reputadas en el ámbito de la investigación de la comunicación, como Nieman Foundation, Kinght Foundation o European Journalism Center, entre otras, señalan el Periodismo de Datos (PD en adelante) como una de las tendencias actuales y de futuro para la profesión periodística. En pocos años el desarrollo y visibilidad de esta modalidad ha aumentado considerablemente y son muchos los medios que cuentan con equipos y espacios específicos de PD.

Esta nueva modalidad de periodismo es una disciplina compleja en torno al a cuál no existen definiciones únicas; en términos generales el PD consiste en tratar y ana- 
lizar grandes bases de datos para generar historias útiles para los ciudadanos. La red y las nuevas herramientas informáticas multiplican exponencialmente la cantidad de fuentes informativas, sobre todo, si existe una ley de acceso a la información pública. El ingente volumen de datos (big data) almacenados y disponibles en internet se convierten en material, dónde buscar, seleccionar, procesar, analizar, comparar y finalmente publicar por parte del periodista. Se trata de un concepto amplio y que cómo bien señala la investigadora y docente del Knight Center, Sandra Crucianelli (2012 a) "cualquier cosa que se diga hoy del periodismo de datos no es definitiva", aludiendo a su incipiente desarrollo.

Son muchos los medios internacionales que cuentan con espacios específicos de esta especialidad y cómo señalan Peiró y Guallar (2013: 29) EEUU el lugar donde se pueden encontrar más muestras. Así son varios los diarios que mediante blogs, secciones de 'Datos' específicas o reportajes incluidos dentro de la sección de multimedia o gráficos trabajan esta especialidad. Por ejemplo, el 'Data Desk' Times, el denominado 'Data Post'2 de The Washintong Post, o el blog 'The Upshot' ${ }^{3}$ publicado por The New York Times. Fuera de los medios en Estados Unidos, podemos citar FiveThrityEight ${ }^{4}$, un blog de datos creado por Nate Silver y que se publicó durante años en The New York Times, hoy convertida en una web independiente de PD con varias secciones y una plantilla de 20 personas.

En Latinoamérica también encontramos algunos ejemplos y quizás el más notorio sea el blog 'Lanación.data' ${ }^{5}$ del diario argentino La Nación. Existen otras experiencias como por ejemplo, la unidad de datos ${ }^{6}$ del diario El Tiempo de Colombia dirigida por Ginna Morelo. También en el periódico La Nación de Costa Rica cuentan con una sección, 'Data'7, para la práctica de esta disciplina.

En Europa el referente es el 'Datablog' ${ }^{8}$ de The Guardian, uno de los primeros medios en poner en marcha una sección específica de esta disciplina; al igual que la corporación $B B C$ cuyos productos de PD se realizan en su 'Lab'. También el diario alemán Zeit Online ha realizado varios proyectos publicados en su sección 'Datenjournalismus' ${ }^{10}$. El diario francés Le Monde mantiene un blog 'J'ai du bon datos' en su versión digital dedicado a datos y visualizaciones dirigido por Alexandre Léchenet.

Pero el PD no sólo tiene cabida en los medios. Existen algunas aplicaciones, plataformas, webs, o fundaciones cuya labor puede ser enmarcada en este ámbito. En el ámbito internacional, cabe destacar, la plataforma colaborativa de PD Poderope-

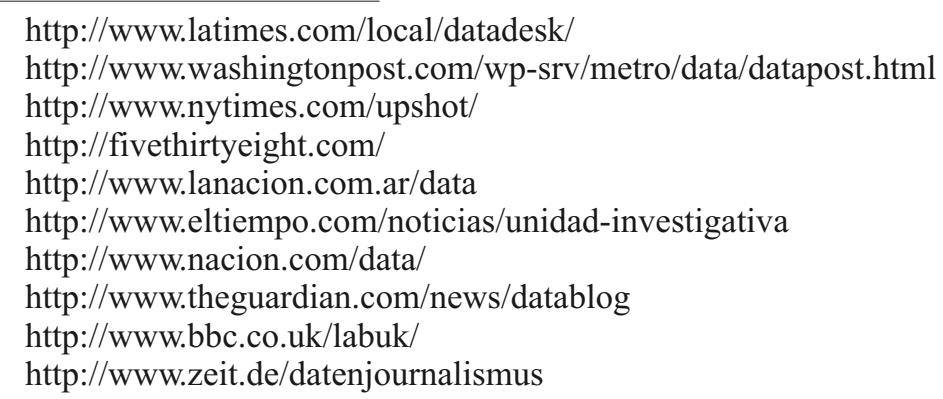


dia.org ${ }^{11}$, el movimiento conocido como 'Chicas Poderosas' iniciado por Mariana Santos ${ }^{12}$ para formar mujeres en tecnología en las salas de redacción en toda América Latina. Para ello enseña, cómo limpiar los datos, cómo hacer visualizaciones, etc.... También la organización sin ánimo de lucro Propublica ${ }^{13}$ (E.E.U.U.) dedicada al periodismo de investigación trabaja esta disciplina. En Europa encontramos, entre otras, Dataninja $^{14}$, una red de PD creada en Italia. Fundada en 2012 por Alessio Cimarelli y Mauro Andrea Nelson ha colaborado con algunos grupos editoriales europeos (Grupo L'Espresso, RCS, Le Monde, etc....). En Francia destaca Journalism $++{ }^{15}$ una red de empresas dedicada al análisis y visualización de datos.

Autores como Chaparro (2013: 7), Ferreras (2013: 130), Peiró y Guallar (2013: 35) señalan que el PD es una especialización poco explotada todavía en España. En los últimos meses se observa un mayor interés por dicha disciplina por parte de las empresas periodísticas, tanto es así que medios como Elconfidencial.com o Elmundo.es han lanzado una sección específica. También los profesionales comienzan a conocer esta modalidad de periodismo; según el Informe Anual de la Profesión Periodística (2013:51) "el porcentaje de profesionales que conocen en mayor o menor grado la existencia del PD ha aumentado en más de 10 puntos porcentuales (con respecto a 2012), hasta el $54,7 \%$, si bien es verdad que todavía son pocos los que trabajan en esta especialidad (6,6\%)".

Por otra parte, existen en España también organizaciones y fundaciones, al margen de los medios, que han creado proyectos o aplicaciones derivadas de dicha modalidad periodística; es el caso, por ejemplo, de la Fundación Civio. Algunos de sus proyectos más conocidos son 'El indultometro' ${ }^{16}$, 'España en llamas'17 o 'Dónde van mis impuestos ${ }^{18}$.

El objetivo principal de esta contribución es establecer una radiografía de la implantación del PD en España; tanto dentro como fuera de los medios, ya que, cómo se ha subrayado esta modalidad no sólo está presente en los medios. A pesar de que se

11 Poderopedia fue lanzado en diciembre de 2012 por el periodista Miguel Paz, ganador de la Beca Internacional de Periodismo Knight, para informar sobre la red de personas, empresas y organizaciones influyentes dentro de su país. Paz y el desarrollador de web Héctor Vergara crearon el proyecto con ayuda de la John S. and James L. Knight Foundation, tras ganar el premio Knight News Challenge de 2011. Es una plataforma sin ánimo de lucro que utiliza información pública de bases de datos gubernamentales y de medios de comunicación. Cuenta también con extensiones en Colombia y Venezuela. http:/www.poderopedia.org/.

12 Fundadora de este movimiento y diseñadora. Trabajó en el departamento de visualización de The Guardian. Ha obtenido una beca del International Center for Journalism y organiza eventos para formar a mujeres en el ámbito del Periodismo de Datos en Latinoamérica.

$13 \mathrm{http}: / /$ www.propublica.org/

14 http://www.dataninja.it/

15 http://www.jplusplus.org/fr/

$16 \mathrm{http} / / / \mathrm{www}$. elindultometro.es/index.html

$17 \mathrm{http}: / /$ www.espanaenllamas.es/

18 http://dondevanmisimpuestos.es/ 
trata de una disciplina en desarrollo, parece adecuado realizar un estudio exploratorio que ofrezca una panorámica de su estado actual en España. Así, los objetivos concretos del mismo son;

1. Identificar los medios de comunicación, empresas o instituciones que publican online trabajos enmarcados en esta disciplina.

2. Comprobar su grado de implantación. Para ello, en primer lugar, es necesario determinar si cuentan o no con equipos específicos de PD. En segundo lugar, conocer las características de estos; miembros, formación, trayectoria y si colaboran con otras instituciones en la realización de trabajos de PD.

3. Percepción de los profesionales sobre el desarrollo de esta disciplina en España

\section{Material y método}

Con el fin de alcanzar estos objetivos se estableció un planteamiento metodológico dual; teórico y práctico. Así, se realizó la pertinente revisión bibliográfica para conocer el contexto en el que se desarrolla el PD. Por otra parte, el planteamiento práctico o trabajo de campo se abordó en dos etapas diferenciadas. Durante la primera fase la autora identificó los medios de comunicación e instituciones que trabajan actualmente esta disciplina en España y cuyos trabajos se publican en Internet. Para ello, además de la consabida observación, se repasaron otros materiales, como publicaciones, jornadas ${ }^{19} \mathrm{o}$ conferencias ${ }^{20}$. En el caso de los medios se tuvieron en cuenta aquellos que trabajan el PD desde sus propias redacciones y también aquellos que han publicado de manera puntual piezas elaboradas por otras instituciones o en colaboración con las mismas. No se hizo distinción en cuanto a la frecuencia de publicación, ni tampoco en cuanto al tipo de medio. Si bien es cierto que esta modalidad parece, al menos a priori, que tiene mayor aceptación en la prensa digital, se revisaron también webs de televisión y de radio. Por otro lado, se identificaron también una serie de empresas o instituciones que practican PD. Esta fase se llevó a cabo durante los meses de junio, julio, agosto y septiembre de 2014.

19 La autora revisó el material publicado en torno a las I Jornadas de Periodismo de Datos y Open Data celebradas del 24 al 26 de mayo de 2013 en Madrid y Barcelona como sedes principales. También segunda edición: II Jornadas de Periodismo de Datos y Open Data, que tuvieron lugar del 24 al 27 de abril de 2014 en Madrid, Barcelona y Almería. Ambos eventos fueron organizados por el grupo local de la Open Knowledge Foundation en España y coordinadas por las periodistas Mar Cabra y Karma Peiró. Véase: http://periodismodatos.okfn.es/. Asimismo se repasaron los contenidos publicados por el Grupo de Trabajo de Periodismo de Datos del MediaLab-Prado en funcionamiento desde el otoño de 2011. Véase: http://medialab-prado.es/article/periodismo_de_datos_-grupo_de trabajo

20 Las conferencias revisadas pertenecen al Festival Internacional de Periodismo 2014 , que se celebra en Perugia. Concretamente a las dictadas en el panel 'Data Journalism', cuyos vídeos estaban disponibles en: http://www.journalismfestival.com/programme/2014/category/data-journalism-school

Por otro lado, el Centro de Cultura Contemporánea de Barcelona (CCCB), también cuenta con un grupo de trabajo sobre Periodismo de Datos, que ya ha mantenido varias sesiones de trabajo y talleres. Disponibles en: http://blogs.cccb.org/lab/es/category/proj/periodismo-datos/ 
En la segunda fase se abordaron los objetivos 2 y 3 . Ambos de marcado carácter profesional, por lo que para su conocimiento no bastaba con la observación o revisión del material publicado; sino que era aconsejable acudir a la fuente. Por ello, se diseñaron los siguientes cuestionarios con preguntas abiertas para los medios, empresas o instituciones que conforman la muestra.

\begin{tabular}{|c|}
\hline $\begin{array}{l}\text { Tabla 1. Cuestionario elaborado para los } \\
\text { medios }\end{array}$ \\
\hline $\begin{array}{l}\text { ¿Cuentan en su redacción con equipo específico } \\
\text { de Periodismo de Datos? }\end{array}$ \\
\hline $\begin{array}{l}\text { Características del equipo: miembros, } \\
\text { formación, trayectoria... }\end{array}$ \\
\hline $\begin{array}{l}\text { ¿Colaboran o han colaborado con otras } \\
\text { instituciones para elaborar informaciones de } \\
\text { PD? }\end{array}$ \\
\hline $\begin{array}{l}\text { Razones por las que el PD, hasta ahora, ha } \\
\text { tenido escasas manifestaciones en los medios } \\
\text { españoles }\end{array}$ \\
\hline $\begin{array}{l}\text { Perspectivas de futuro de esta disciplina en } \\
\text { España }\end{array}$ \\
\hline
\end{tabular}

\begin{tabular}{|l|}
\hline \multicolumn{1}{|c|}{$\begin{array}{c}\text { Tabla 2. Cuestionario elaborado para } \\
\text { empresas e instituciones }\end{array}$} \\
\hline $\begin{array}{l}\text { ¿Cuenta su institución con equipo específico de } \\
\text { Periodismo de Datos? }\end{array}$ \\
\hline $\begin{array}{l}\text { Características del equipo: miembros, formación, } \\
\text { trayectoria... }\end{array}$ \\
\hline $\begin{array}{l}\text { ¿Colaboran o han colaborado con medios de } \\
\text { comunicación en la realización de informaciones } \\
\text { de PD? ¿Y con otras instituciones? }\end{array}$ \\
\hline $\begin{array}{l}\text { Razones por las el PD, hasta ahora, ha tenido } \\
\text { escaso desarrollo en España }\end{array}$ \\
\hline $\begin{array}{l}\text { Perspectivas de futuro de esta disciplina en } \\
\text { España }\end{array}$ \\
\hline
\end{tabular}

Se trata de un estudio, exploratorio y descriptivo que debe ser entendido como punto de partida para otras investigaciones posteriores.

\section{Periodismo de Datos: nueva modalidad informativa}

Ciertamente el periodismo siempre ha utilizado el análisis de datos para informar, contextualizar, elaborar reportajes... Es decir, no es nada nuevo, como señalan Flores y Cebrián (2012:221), Crucianelli (2012 a), Rogers (2012: 20) incluso a finales del siglo XIX encontramos en periódicos impresos ejemplos de la utilización de datos para elaborar informaciones más completas ${ }^{21}$. Pero en los últimos años estamos asistiendo al desarrollo y auge de un nuevo método periodístico basado en el análisis de grandes volúmenes de datos; apoyado en potentes herramientas que procesan los datos y que involucra a otros profesionales como, programadores, estadísticos o diseñadores.

Se trata de una modalidad aún en desarrollo, lo que explica que su conceptualización no sea única y que existan diferentes definiciones en torno al PD. Flores y Cebrián (2012: 222) entienden que el PD es una forma de periodismo de investigación que utiliza la informática para buscar, contrastar, visualizar, combinar datos y proporcionar información de diferentes fuentes, públicas o privadas.

Por su parte, Peiró y Guallar (2013: 26) consideran que PD es "la especialidad o práctica del periodismo que utiliza como materia prima y fundamental datos (y no otras fuentes de información), a fin de crear productos informativos diversos, tales como: artículos con datos, infografías o visualizaciones interactivas con datos en abierto. El énfasis de la definición está claramente en el término 'datos'. Estas son la fuente y el origen de la información que se publica y son también las protagonistas destacadas del producto informativo que se ofrece al lector".

21 Simon Rogers, editor del Datablog del diario The Guardian, detalla en su libro Facts are sacred varios ejemplos aparecidos en la prensa inglesa a principios del siglo XX. 
Paul Bradshaw ${ }^{22}$ (2012), gran impulsor de esta disciplina, considera que el PD se diferencia del resto por "las nuevas posibilidades que se abran cuando se combina el tradicional olfato periodístico y la capacidad de contar una historia convincente, con la magnitud y el alcance de la información digital disponible".

Para comprender la evolución de esta modalidad es necesario revisar sus antecedentes que autores como, Crucianelli (2012 a), o Bounegru (2012:20) los sitúan en el llamado Periodismo de Precisión, término utilizado para clasificar artículos en los que se aplicaba el método científico. Su despegue definitivo vendría de la mano del periodista y profesor Philip Meyer con la publicación de su libro Precision Journalism. En síntesis, se trata de la aplicación de métodos científicos de las ciencias sociales a la práctica periodística.

A medida que la tecnología evolucionaba fue posible rastrear y analizar bases de datos con el fin de contextualizar hechos informativos; y comienza a hablarse de $d a$ tabase journalism, Computer-Assisted Journalism (CAJ) o de CAR (Computer-Assisted Reporting); modalidad del periodismo de precisión en la que se utilizan los ordenadores para examinar las bases de datos y descubrir asociaciones o correlaciones estadísticas en todo tipo de documentos informatizados. Los antiguos conceptos de CAJ y CAR, utilizados sobre todo en el Reino Unido y en Estados Unidos a finales de los años 90, se referían al uso de ordenadores en la creación de contenidos. En ese concepto se tenía en cuenta al ordenador para obtener ideas de cara a elaborar noticias, y se usaba el software (minería de datos) para tratar datos. Pero no iba más allá, simplemente por la fase primigenia en la que se encontraba la red.

Lo que se conocía como CAR o CAJ no parece diferenciarse significativamente, a priori, de lo que hoy se conoce como PD; de modo que ha surgido un debate en torno a la novedad de esta disciplina. Sin embargo y siguiendo a Meyer, existe una diferencia fundamental: la abundancia de información. Así lo reconoce en The Data Journalism Handbook (2012: 6) "Cuando la información era escasa, la mayor parte de nuestros esfuerzos se dedicaron a la caza y la recolección. Ahora que la información es abundante, el procesamiento es más importante". Es decir, la novedad ahora reside en el ingente volumen de datos (big data), en muchas ocasiones públicos, almacenados y disponibles en Internet que a su vez se convierten en material, dónde buscar, seleccionar, procesar, analizar, comparar y finalmente publicar por parte del periodista. Y también en las tecnologías y programas que hoy permiten desarrollar dicho proceso desde el principio al fin.

Liliana Bounegru, del European Journalism Center (EJC), mantiene una posición más ecuánime al respecto y argumenta: "En lugar de debatir si el PD es completamente nuevo, sería más fructífero considerarlo como parte de una tradición más larga, pero respondiendo a las nuevas circunstancias y condiciones. Aunque puede que no

22 Profesor y director del Máster en Periodismo Digital de la Birmingham City University. Es el editor del blog Online Journalism Blog, y el fundador del sitio web de periodismo de investigación HelpMeInvestigate. Ha escrito para los blogs de datos de los medios británicos The Guardian y The Telegraph. Se pueden consultar en su blog: http://onlinejournalismblog.com/books/ 
haya una diferencia de objetivos y técnicas, la aparición de la etiqueta de 'periodismo de datos' a principios del siglo indica una nueva fase en la que el volumen de datos que está disponible gratuitamente en línea en combinación con sofisticadas herramientas centradas en el usuario, la auto-publicación y herramientas crowdsourcing permite a más gente a trabajar con más datos con más facilidad que nunca" (Bounegru, 2012: 21).

Podemos, por tanto, calificar el PD como una nueva modalidad o disciplina informática en la que se dan tres elementos fundamentales; acceso a los datos, su tratamiento y su publicación; cuyo denominador común son las nuevas herramientas y tecnologías. Elementos que conforman, a su vez, las fases de trabajo en esta disciplina. Precisamente Bradshaw publicó en $\operatorname{su}_{\text {blog }}{ }^{23}$ un interesante diagrama, con forma de pirámide invertida, para representar las diferentes fases del proceso de producción del PD.

Imagen 1. Pirámide invertida de Periodismo de Datos.

Fuente: http://onlinejournalismblog.com

The Inverted Pyramid of Data Journalism

Paul Bradsahw: onlainejournalismBlog.com

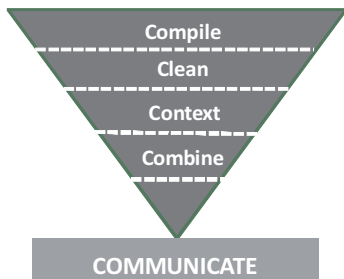

COMMUNICATE

La compilación de los datos alude directamente a las fuentes informativas. En este sentido, existen dos tipos de fuentes; públicas ${ }^{24} \mathrm{y}$ privadas. Se puede acceder a ellas mediante peticiones de información, búsquedas avanzadas o con técnicas de scraping (rascar). También es posible obtener datos tomando información de APIs o a través de encuestas, formularios online o crowdsourcing. Pero además, será necesario revisar la calidad y unificar formatos (limpiar datos), por ejemplo, con programas como Google Refine. La contextualización está asociada con la interpretación de los datos y con la combinación con otros que permita completarlos y comprender su relevancia. Así, es frecuente contextualizar y combinar datos con geolocalizaciones y mapas.

Por último, estaría la fase de comunicación; es el momento de exponer los resultados y son muchas las formas de hacerlo. Quizá las más conocidas sean las visualizaciones de datos, pero también se pueden utilizar textos, aplicaciones (mashup), mapas, etc.... En esta etapa, autores como Rodríguez y García, (2014: 60), Zanchelli y Crucianelli (2012:4), Gordon (2007) y profesionales como Daniele Grasso (Elcon-

23 http://onlinejournalismblog.com/2011/07/13/the-inverted-pyramid-of-data-journalism-part2-6-ways-of-communicating-data-journalism/

24 En el ámbito público España cuenta con una Ley de Transparencia aprobada en diciembre de 2013. Además algunas comunidades autónomas como País Vasco o Aragón cuentan con plataformas de datos abiertos dónde puede encontrarse información reutilizable. 
fidencial.com) o Mar Cabra ${ }^{25}$ (ICIJ) consideran imprescindible trabajar con equipos multidisciplinares: diseñadores, arquitectos de información y programadores que junto con los periodistas consigan elaborar piezas basadas en grandes volúmenes de datos de manera clara y eficaz. Además, sostienen la conveniencia de publicar los datos brutos y explicar las metodologías utilizadas para fomentar la transparencia.

Por otro lado, conviene subrayar que desde el punto de vista teórico así como desde la perspectiva más profesional son muchas las iniciativas que han contribuido a la evolución de esta modalidad. En el plano teórico destacan algunas publicaciones, por ejemplo, el artículo 'Data as journalism, journalism as data' que el profesor Gordon publica en 2007 en el que explica que cuando se unen el reportaje tradicional con las nuevas capacidades de uso de las bases de datos, surgen nuevas formas de periodismo, que se implantarán gracias a la aparición de herramientas tecnológicas más sencillas y baratas.

También el video documental "Journalism in the age of data" (2010) producido por el periodista Geoff McGhee para la universidad de Standford, dónde asegura que ante la ingente cantidad de datos que se generan cada día la labor del periodista es "organizar, dar sentido y sintetizar la masiva oleada de información en bruto que el auge de la comunicación digital propicia, pero también diseñar herramientas para que los lectores completen dichas operaciones por sí mismos".

Por su parte, Simon Rogers ${ }^{26}$, encargado del Data Store de The Guardian hasta 2013, publicó Facts are Sacred: The Power of Data (2011) dónde explica el proceso de trabajo en el diario y desafíos para encontrar la información y visualizarla. Además, asegura que el PD no es nada nuevo pero esta vez llegó para quedarse puesto que ya es un estándar de la industria. Ya en 2012 aparece The Data Journalism Handbook ${ }^{27}$ una iniciativa de la Open Knowledge Foundation y el European Journalism Centre. En él colaboran expertos en PD de medios, universidades y blogs de todo el mundo. En 2014 se publicó el Manual de Periodismo de Datos Iberoamericano, con participación de expertos y periodistas de Latinoamérica, España y Portugal.

Del mismo modo, en el plano profesional o práctico se han sucedido iniciativas que también han contribuido al avance y promoción de esta disciplina. Así, en 2007 la Knight Fundation premió el proyecto de Adrien Holovaty, llamado EveryBlock, una web que permite conocer a los usuarios información relativa a su barrio obtenida de bases de datos. Por otro lado, en 2009 el periódico norteamericano St. Petersburg Times recibió el premio Pulitzer por el proyecto Politi Fact. Un entorno web donde se analizaban las declaraciones de políticos para evaluar el grado de verdad.

En agosto 2010 el Centro Europeo de Periodismo organizó en Amsterdam la primera Conferencia sobre Data Journalism. Por otro lado, en 2011 los 16 proyectos ganado-

25 Periodista especialista en datos y miembro del Consorcio Internacional de Periodistas de Investigación (ICIJ). Fundadora de la Fundación Ciudadana Civio, fue su directora hasta diciembre de 2012. Es profesora de Periodismo de Datos en el Máster de Periodismo de Investigación de Unidad Editorial y de Periodismo Digital del Instituto de Empresa.

26 Estuvo al frente del Data Store en The Guardian hasta 2013. Actualmente es el Data Editor de Twitter.

27 Desde abril de 2013 está disponible en castellano gracias a la traducción realizada por el equipo de PD de La Nación (Argentina). 
res del Knight News Challenge, un concurso anual de la Knight Foundation destinado a promover la innovación en el ámbito del periodismo, tuvieron al Periodismo de Datos como denominador común. En 2012 la Knight Foundation, Google y la Red Global de Editores (GEN) pusieron en marcha los premios 'Data Journalism' con el propósito de destacar el valor del mismo a editores y ejecutivos de medios. Su última edición se celebró en Barcelona dentro del evento GEN Summit 2014 y fueron premiadas piezas de PD de Pro Publica, The New York Times o La Nación entre otros.

En Europa destaca The International Journalism Festival ${ }^{28}$ un macroevento que se celebra desde 2006 en Perugia (Italia) y que cuenta con innumerables talleres y conferencias, entre ellos, 'The school of Data Journalism'29 impulsado por el European Journalism Center $^{30}$ y The Open Knowledge Foundation ${ }^{31}$. En España las I y II Jornadas de Periodismo de Datos y Open Data organizadas por la OKFN Spain (Open Konwledge Foundation), así como los grupos de trabajo creados en Media-Lab Prado, o en el Centro de Cultura Contemporánea de Barcelona han contribuido a la promoción del PD.

\section{Análisis y resultados}

\subsection{Identificación de la muestra}

Cómo vemos en el cuadro serían 14 los medios que publican online contenido enmarcado en el PD y dos las instituciones; una empresa y una fundación. Conviene resaltar algunas particularidades de la muestra. Lainformación.com, probablemente fue uno de los medios pioneros en la publicación de visualizaciones basadas en datos, pero, desde 2013 parece tener paralizada su sección de gráficos ${ }^{32}$. Llama la atención la presencia de medios regionales online como Vilaweb e iLeón y Diario de Navarra, este último sí tiene versión papel. También presentes medios relativamente nuevos como Acuerdo y Eldiario.es. En cuanto a las cabeceras más tradicionales encontramos Elpais.com, Elmundo.es. 20minutos.es y Elperiodico.com mientras que el plano audiovisual se identificaron tres medios; Rtve.es, Cadenaser.com y Tv3.cat.

Tabla 3. Muestra identificada. Fuente: Elaboración propia

\begin{tabular}{|c|}
\hline Medios de Comunicación \\
\hline Rtve/lab, Elconfidencial.com, El Periódico de Catalunya, 20 Minutos, El País, \\
El Mundo, Vilaweb, Diario de Navarra, Lainformación.es, iLeón/lab, Acuerdo, \\
Cadena Ser, Tv3, Eldiario.es \\
\hline \hline Empresas / Instituciones \\
\hline Fundación Civio, Data'n'Press \\
\hline
\end{tabular}

28 http://www.journalismfestival.com/

29 Se trata de una serie de talleres, paneles y conferencias en torno al Periodismo de Datos. La primera edición tuvo lugar en 2012 en el marco del International Journalism Festival y desde entonces se han celebrado otras dos citas en 2013 y 2014.

30 Centro Europeo para el Periodismo. Se puede consultar su web en: http://www.ejc.net/ejc/

31 Organización sin ánimo de lucro que trabaja para el fomento del conocimiento abierto y por el acceso a la información. Se puede consultar en: https://okfn.org/

32 Esta era la sección donde se publicaban dichas visualizaciones basadas en datos y según aparece en su web la última publicada data de 2012. Se puede comprobar el archivo de dichos gráficos en: http://graficos.lainformacion.com/ 
En el capítulo de instituciones se registraron dos; la Fundación Civio y la empresa Data'n'Press. La primera, es una entidad sin ánimo de lucro constituida a finales de 2011. Su trabajo se centra en el fomento de la transparencia y en la promoción del acceso a datos públicos por parte de cualquier ciudadano u organización. Para ello, desarrollan herramientas y aplicaciones que faciliten el acceso y análisis de la información pública y muchos de sus proyectos se materializan utilizando el PD. Algunos de los más conocidos serían 'España en llamas', 'Tu derecho a saber', o 'El Indultométro'. Las donaciones ciudadanas son su principal fuente de financiación.

Data'n'Press, por su parte, se define como estudio de Periodismo de Datos y análisis de Big Data. Fundado por el informático Arnau Udina ${ }^{33}$, David Martín-Borregón $^{34}$, y Eduard Martín-Borregón ${ }^{35}$ a finales de 2011. Muchos de sus trabajos hasta ahora se han centrado en el análisis de datos procedentes de la red de microblogging Twitter, como 'Twitterencatala.org' ${ }^{36}$, ‘@ resultados $20 \mathrm{~N}^{37}$. También han colaborado con medios de comunicación como veremos más adelante.

\subsection{Implantación del Periodismo de Datos}

\subsubsection{Análisis de los medios de comunicación}

No todos los medios contestaron el cuestionario; no se obtuvo respuesta ${ }^{38}$ del mismo por parte de Elmundo.es, Elpais.com, Lainformación.es, Vilaweb y Eldiario.es. A pesar de la merma que esto puede suponer para el estudio, el resto de medios e instituciones aportaron gran cantidad de información, suficiente para comprobar el grado de implantación del Periodismo de Datos en España, dado que se trata de un estudio de carácter exploratorio. Así, los datos recabados fueron proporcionados por Miriam Hernanz (Rtve.es/lab), Daniele Grasso (Elconfidencial.com), Elisa Vivas (Elperiodico.com),

33 Informático con dilatada experiencia en medios comarcales.

34 Ingeniero superior en Telecomunicación, Ingeniero técnico en imagen y sonido, Máster en Inteligencia Artificial y Biga Data por la Universidad Pompeu Fabra.

35 Periodista y Máster en Periodismo de Datos, Investigación y Visualización de Datos (URJC) con beca Google a la Excelencia. También ha cursado el primer ciclo de Economía en la Universidad Autónoma de Barcelona.

36 Aplicación que realiza un recuento de los usuarios que modifican su interface de Twitter al catalán, de julio de 2012. En septiembre con motivo del 11 de septiembre se incorporó un recuento de los hashtags más usados en Twitter durante la Diada. Se pude ver en: http://twitterencatala.org/

37 Se puede consultar en el siguiente blog: http://resultados20n.blogspot.com.es/

38 La autora se puso en contacto con todos los medios e instituciones recogidas en la muestra a través de correo electrónico, mensajes en Facebook y Twitter. Vilaweb.cat, Eldiario.es y Lainformación.es no respondieron. Desde Elpais.com, su directora de Producto Digital de Prisa, indicó que no cuentan con unidad de datos como tal y que solamente publican alguna pieza de forma puntual. Por su parte desde el diario Elmundo.es se señaló a mitad de julio que "la unidad de periodismo de datos como tal está en proceso de creación y arrancará en breve". La autora observó que desde agosto se publica la sección Elmundo.datos (http://www.elmundo.es/especiales/datos/) con piezas de Periodismo de Datos, y de nuevo se puso en contacto con el periódico, pero no obtuvo respuesta. Así, la información relativa a estos medios que se ofrece en la contribución procede de la observación. 
Idioa Sota (Acuerdo), Virginia Pérez (20minutos.es), Ramaris Albert (diariodenavarra.es), Jesús Uribe (iLeon.es), Jon Laiseca (Cadenaser.com), Judith Argilas (TV3.cat), Eduard Martín (Data'n'Press) y Eva Belmonte (Fundación Civio).

Así, una vez codificados los resultados comprobamos que cinco medios cuentan con equipos específicos; tres publican contenido de PD desde otros departamentos y dos más estarían en proceso de lanzar una sección específica.

Tabla 4. Medios y equipos. Fuente: Elaboración propia

\begin{tabular}{|c|c|c|}
\hline Equipo específico & Dpto. Infografía /Otros & En proceso \\
\hline Elconfidencial.com & Elperiodico.es & Diariodenavarra.es \\
\hline Elmundo.es & 20Minutos.es & Acuerdo \\
\hline Rtve/Lab & Cadenaser.com & \\
\hline iLeon.es & Tv3.cat & \\
\hline
\end{tabular}

Si bien en la muestra aparecen más medios, en el cuadro sólo se codifican aquellos que han respondido al cuestionario ${ }^{39}$, excepto el diario Elmundo.es puesto que en su web sí aparece sección de datos. En el caso de Elpais.com tampoco se codificó, dado que Noemí Ramírez, Directora de Producto Digital de Prisa confirmó que este diario no cuenta con equipo específico de PD. Sin embargo, no podemos dejar de subrayar que sí ha publicado piezas puntuales como la investigación denominada 'Chinaleaks ${ }^{40}$, realizada por el Consorcio Internacional de Periodistas de Investigación $(\mathrm{ICIJ})^{41}$ y publicada a principios de 2014 por varios medios, como Le Monde, BBC o The Guardian.

La sección de PD del diario Elconfidencial.com se integra en el denominado 'Lab' y está formada por Jesús Escudero y Daniele Grasso ambos periodistas y ex alumnos del Máster en Periodismo de Investigación, Datos y Visualización de Unidad Editorial y la Universidad Rey Juan Carlos. Además para elaborar las visualizaciones cuentan con el apoyo de los diseñadores David Gutiérrez y Pablo López. Por otro lado, cabe mencionar que su proyecto 'The migrants Files' ${ }^{42}$ lanzado a principios de 2014 obtuvo el premio Data Journalism Awards en la categoría 'Data Stories on a Single Topic'.

En el caso de Elmundo.es cómo se ha apuntado anteriormente, este diario no respondió al cuestionario; sin embargo, desde este verano cuenta con una sección denominada ElMundo.Datos ${ }^{43}$ cuyas piezas son realizadas por alumnos o ex alumnos (ahora becarios en el diario) del Máster de Periodismo de Investigación, Visualización y Datos que imparten dicho diario y la Universidad Rey Juan Carlos.

Por su parte, RTVE cuenta con una sección de PD integrada en el Laboratorio de Innovación Audiovisual compuesto por dos redactores, dos realizadores, un diseñador

\footnotetext{
39 No aparecen Vilaweb, Eldiario.es ni Lainformación.es

40 Se trata de una investigación que revela actividades económicas en paraísos físcales de empresas y dirigentes chinos. Se puede consultar en: http://elpais.com/tag/chinaleaks/a/

41 http://www.icij.org/

42 Véase: http://www.elconfidencial.com/tags/temas/the-migrants-files-10249/

43 Véase: http://www.elmundo.es/especiales/datos/
} 
y tres desarrolladores. En las piezas de PD trabajan habitualmente, un redactor, un desarrollador y un diseñador. En 2011 publicaron la primera pieza de PD; una narrativa sobre las elecciones autonómicas y los programas de los diferentes partidos políticos ${ }^{44}$.

El diario digital regional iLeon.es lanzó su primer proyecto en mayo de 2014; con varias piezas acerca de las elecciones europeas en León ${ }^{45}$. Según describe Jesús López de Uribe, el equipo de PD está formado por un periodista, un periodista-economista, un informático y un infógrafo. Su intención es continuar publicando trabajos de PD sobre la situación económica de la provincia de León.

Por otro lado estarían los medios que no cuentan con secciones específicas de PD, pero publican piezas basadas en datos desde otros departamentos, como Infografía. Sería el caso de Elperiodico.com, 20Minutos.es, Cadenaser.com y Tv3.cat.

En Elperiodico.com, cómo ya señala Bueno (2014:33), no existe sección de Periodismo de Datos. Los trabajos basados en tratamiento de amplios volúmenes de datos están integrados dentro de la sección de Infografía del diario. La visualización titulada 'El tercio Socialista de Barcelona' ${ }^{46}$ de diciembre de 2012 fue la primera presentada por este diario. Merece la pena destacar su proyecto 'Fuga2'47, sobre la marcha de investigadores españoles al extranjero, cuyos datos se ofrecen en diversos mapas interactivos que muestran la ubicación de dichos investigadores. Esta pieza ganó el segundo premio Innnova Data promovido por la Fundación BBVA en 2013 y se financió con ese dinero. Fue creada por los periodistas Elisa Vivas, Francisco J. Moya y Michele Catanzaro.

Tampoco el diario 20Minutos.es cuenta con una unidad de datos como tal; sino con un departamento de Infografía para la versión digital compuesto por una persona, con formación en comunicación y diseño. Situación similar a la de Cadenaser.com cuyos trabajos hasta ahora han sido elaborados por el redactor jefe de la web, Jon Laiseca; quién además subraya que la redacción está recibiendo formación en esta disciplina.

En la corporación pública Tv3.cat los proyectos basados en datos se trabajan desde las redacciones digitales y desde los equipos de desarrollo de producto digital ya que no existe un equipo de PD específico. La primera pieza que se elaboró en los Medios Digitales de la CCMA (Corporación Catalana de Medios Audiovisuales) que incluye las redacciones digitales de TV3, 324, ESPORT3 y CatRàdio fue 'El Tuitòmetre de les eleccions' ${ }^{48}$, en octubre de 2012 en el que se analizaba el discurso público en Twitter alrededor de las elecciones autonómicas de dicho año.

Además, Diariodenavarra.es y Acuerdo, manifiestan encontrarse en proceso de lanzar secciones propias de PD. En el caso del diario regional, se integraría en el llamado Laboratorio de Innovación Periodística creado por este diario en 2013. De

44 Véase en: http://www.rtve.es/noticias/elecciones/autonomicas-municipales/programaselectorales/

45 Véase en: http://www.ileon.com/politica/040510/proyecciones-electorales-en-la-provinciamirando-a-las-municipales-de-2015-de-reojo y también en http://www.ileon.com/politica /040489/lo-mas-curioso-del-voto-de-las-europeas-en-los-municipios-de-la-provincia-de-leon

46 Véase: http://www.elperiodico.com/es/noticias/politica/tercio-socialista-barcelona-2264103

47 Véase: http://data.elperiodico.com/

48 Véase: http://www.324.cat/eleccions-catalunya-2012/tuitometre 
hecho, varios de sus miembros han participado ya en diversas actividades de formación en PD y visualización de datos, como se puede ver en su blog ${ }^{49}$.

En cuanto a las colaboraciones, encontramos varios medios que han cooperado con entidades externas en la creación de piezas de $\mathrm{PD}$. Al principio de este apartado se ha citado el caso de Elpais.com y el Consorcio Internacional de Periodistas de Investigación (ICIJ). Bien, pues esta organización también ha colaborado Elconfidencial.com, concretamente con la investigación titulada "Offshore Leaks' sobre sociedades paraísos fiscales ${ }^{50}$. Además, esta fue la primera pieza publicada por el equipo de Datos en el citado diario. Igualmente han colaborado con la Fundación Civio, y con la empresa Data'n'Press incluidas en este estudio.

Elperiodico.es, también han trabajado con Data'n'Press, por ejemplo, en el proyecto titulado 'La Mercé geolocalizada' ${ }^{51}$. Asimismo, Vilaweb y Eldiario.es son también clientes de la citada empresa. Este último periódico, en colaboración con el grupo español de la Open Knowledge Foundation (OKF), eligió en mayo de 2013 el proyecto sobre la memoria histórica "Vidas contadas" 52 , como ganador del premio Eldiario.es al mejor proyecto de Periodismo de Datos.

\subsubsection{Análisis de las organizaciones}

Ambas instituciones, Civio y Data'n'Press, como es evidente cuentan con equipos propios y específicos de Periodismo de Datos.

Así en la Fundación Civio cuatro miembros conforman el equipo cuatro personas; David Cabo ${ }^{53}$ (Patrono Fundador y Director), Eva Belmonte ${ }^{54}$ (Responsable de Proyectos), Cristina Moreno ${ }^{55}$ (Alianzas y Fundraising) y Javier de Vega ${ }^{56}$ (Responsable de Comunicación). Además, cuentan con varios colaboradores, Hugo Garrido ${ }^{57}$ (Pe-

$49 \mathrm{http}: / /$ laboratorio.diariodenavarra.es/blog/

50 El contenido de la investigación también fue publicado por varios diarios, entre ellos, The Guardian, Le Monde, los alemanes Suddeustsche Zeitung y Norddeutscher Rundfunk, o The Washington Post. Disponible en: http://www.elconfidencial.com/especiales/paraisosfiscales/

51 Se puede ver en: http://merce2012.elperiodico.com/

52 Se trata de un proyecto elaborado por un grupo de periodistas, programadores y desarrolladores sobre la memoria histórica. Se puede ver en: http://vidascontadas.org/

53 Ingeniero Superior en Informática y Licenciado en Psicología. Ha trabajado como consultor, desarrollador y arquitecto de software. Es el actual director de Civio y entre sus proyectos destacan 'Dondevanmisimpuestos' y 'Tuderechoasaber'.

54 Periodista, ha trabajado en Elmundo.es y su proyecto más destacado es 'El BOE nuestro de cada día' un blog integrado en Civio dónde divulga y analiza las decisiones aparecidas en el Boletín.

55 Licenciada en Ciencias Políticas y Maestría en Geografía Humana y Desarrollo Internacional. Ha trabajado para empresas sociales, organizaciones internacionales y organizaciones no gubernamentales en Latinoamérica, África y Asia, gestionando distintos programas de desarrollo y cooperación.

56 Licenciado en Periodismo, máster en Relaciones Internacionales y especialista en Comunicación e Internacionalización de la empresa española.

57 Ingeniero Técnico en Informática de Sistemas por la Universidad de Alicante. 
riodismo y análisis de datos), Marcos García Rey ${ }^{58}$ (Responsable de 'España en llamas') y Juan Elosua ${ }^{59}$ (Desarrollador). Desde su puesta en marcha en 2011 son varios los proyectos de Periodismo de Datos que han desarrollado; el primero fue '¿Dónde van mis impuestos?' ${ }^{60}$. En marzo de 2012 se lanzó 'Tu Derecho a Saber' 61 , 'El Indultómetro' 62 en febrero de 2013 y 'España en Llamas' ${ }^{63}$ suma dos versiones publicadas en los veranos de 2012 y 2013. Su último proyecto es '¿Quién manda?' ${ }^{64}$, en el que exploran los intereses y relaciones de poder en España. En estos momentos trabajan para poner en marcha '¿Quién cobra la obra?' ${ }^{65}$, proyecto con el que pretenden investigar la contratación de obra civil en España. Para ello iniciaron en octubre de 2014 una campaña de cofinanciación para poder sufragar los gastos.

La Fundación Civio también ha colaborado con otras instituciones para desarrollar algunos de estos proyectos. Por ejemplo, 'Tu Derecho a Saber' se llevó a cabo junto con Acces Info Europe, organización de derechos humanos dedicada a promover y proteger el derecho de acceso a la información en Europa. Y 'Quién manda' se ha realizado con la colaboración de la organización estadounidense Global Integrity. Sin embargo, cómo señala Eva Belmonte, de momento no han colaborado directamente con medios de comunicación, 'aunque están en vías de hacerlo'; pero dado que sus contenidos son Creative Commons algunos medios los republican.

Por último, conviene subrayar su labor de formación en esta disciplina. En 2014 se puso en marcha la Escuela Civio ${ }^{66}$, donde se ofrecen online (previo registro) una serie de recursos sobre cómo y dónde obtener datos, su tratamiento, herramientas útiles en PD, así como sobre transparencia y Open Data. Han impartido formación en el Gobierno del País Vasco, el Grupo Prisa, en el Centro de Innovación BBVA, en el Máster de Periodismo de El País y en el Máster de Periodismo de Investigación, Datos y Visualización de Unidad Editorial y la Universidad Rey Juan Carlos I. También participan en otras actividades como las Jornadas de Periodismo de Datos y Open Data organizadas por la OKFN Spain (Open Konwledge Foundation), en el Grupo de Pe-

58 Miembro del International Consortium of Investigative Journalists. También coordina el Máster en Periodismo de Investigación, Datos y Visualización organizado por Unidad Editorial y la Universidad Rey Juan Carlos.

59 Ingeniero de Telecomunicaciones y trabaja como desarrollador freelance con experiencia en análisis y visualizaciones de datos. Ha colaborado con Civio en los proyectos de periodismo de datos 'España en Llamas' y 'El Indultómetro' e integrado la parte autonómica dentro del proyecto ‘¿Dónde van mis impuestos?'.

$60 \mathrm{http}: / /$ dondevanmisimpuestos.es/

61 http://www.tuderechoasaber.es/

$62 \mathrm{http}: / / \mathrm{www}$. elindultometro.es/index.html

$63 \mathrm{http}: / /$ www.espanaenllamas.es/

64 http://quienmanda.es/

65 http://www.civio.es/2014/10/quien-cobra-la-obra-ayudanos-a-investigar-la-contratacionde-obra-civil/

66 http://escuela.civio.es/ 
riodismo de Datos de MediaLab- Prado ${ }^{67}$ o el Grupo de trabajo de PD del Centre de Cultura Contemporània de Barcelona ${ }^{68}$.

El equipo de Data'n'Press está constituido por sus fundadores, Arnau Udina, David Martín-Borregón, y Eduard Martín-Borregón. Además de los trabajos anteriormente citados, esta empresa ha colaborado con diversos medios de comunicación. En septiembre de 2012 realizó para Elperiodico.es un trabajo titulado 'La Mercé geolocali-

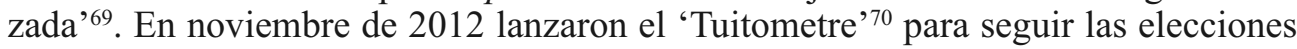
catalanas y "Mapa del Sobiranismo" 71 que muestra los resultados de las elecciones catalanas pueblo por pueblo. Ambos proyectos fueron publicados en Vilaweb, uno de los medios incluidos en este estudio que no respondió el cuestionario planteado por la autora. Por otra parte, Data'n'Press creó una aplicación de resultados en directo de las elecciones europeas ${ }^{72}$ del año 2014 para Elconfidencial.com, también incluido en esta contribución.

Al igual que Civio, han participado en diversas actividades relacionadas con la formación en PD; por ejemplo, en el I Curso de Verano de Periodismo de Datos de la Universidad Complutense en 2012 o en las I y II Jornadas de Periodismo de Datos y Open Data organizadas por la OKFN Spain (Open Konwledge Foundation) en 2013 y 2014 o en el Grupo de Trabajo de PD de MediaLab-Prado.

\subsubsection{Percepción de los profesionales}

Atendiendo a las respuestas obtenidas los profesionales apuntan tres razones fundamentales para en cuanto a la escasa presencia de PD hasta ahora en los medios españoles; falta de recursos económicos, de rentabilidad, y de formación de las redacciones. Todos reconocen que en el contexto de crisis y sin proyectos claros de futuro en Internet no se fomentan las iniciativas de innovación. Sin embargo, se subraya que no "falta conciencia innovadora", sino recursos económicos. Pero también se señala, desde Elconfidencial.com y Elperiodico que "la falta de recursos no debería de ser un freno para el PD puesto que existen muchas herramientas gratuitas y cursos gratuitos de formación". Además, ambos medios, junto con 20minutos.es y Rtve.es destacan que el desarrollo del PD se ha visto lastrado por la organización jerárquica de las redacciones, y por el escaso interés en reciclarse por parte de los periodistas más veteranos.

Por otro lado, entienden que no genera rentabilidad; el PD requiere tiempo, y los medios buscan inmediatez en la generación de contenidos, (Cadenaser.com, Rtve.es/Lab, iLeon.es). Se apunta además la insuficiente formación como otra de las causas que han marcado la evolución del PD en España, así como la necesidad de contar con equipos multidisciplinares (informáticos, diseñadores, desarrolladores...).

67 http://medialab-prado.es/article/periodismo_de_datos_-_grupo_de_trabajo

$68 \mathrm{http} / / / \mathrm{blogs} . c c c b . o r g /$ lab/es/category/proj/periodismo-datos/

$69 \mathrm{http}: / /$ merce2012.elperiodico.com/

$70 \mathrm{http}: / / \mathrm{www} . v i l a w e b . c a t /$ tuitometre

71 http://www.vilaweb.cat/mapa-electoral

$72 \mathrm{http}: / /$ www.elconfidencial.com/elecciones-europeas-2014/ 
En lo referente al futuro del PD, las perspectivas de los profesionales participantes en este estudio preliminar pasan por la formación y por la necesidad de "rediseñar las estructuras en las redacciones apostando por secciones transversales y multidisciplinares" (20minutos.es, Elperiodico.es, iLeón.es, Cadenaser.com).

Mientras, la Fundación Civio y Data'n'Press estiman que es posible que a corto plazo el PD vinculado a los medios se vea lastrado por la falta de recursos, pero entienden que pueden crear proyectos para otras instituciones. Del mismo modo manifiestan que la transparencia y el acceso público a los datos son a día de hoy un desafío para la implantación y estandarización de esta disciplina en España.

\section{Conclusiones}

En esta contribución se ha tratado de establecer una radiografía sobre la situación del Periodismo de Datos en España; tanto dentro como fuera de los medios.

Aunque esta modalidad es considerada como una línea de futuro para la profesión, el uso de sus potencialidades aún es limitado en gran parte de los medios de comunicación en España. Prueba de ello es que sólo se han identificado 14 medios que publican, en muchas ocasiones sólo de forma esporádica, informaciones de PD. Sólo cuatro de los medios identificados cuentan con equipos propios y multidisciplinares en esta disciplina: Elconfidencial.com, iLeón.es, Elmundo.es y Rtve.es/lab; todos ellos puestos en marcha entre 2011 y 2014. Otros, como Elperiódico.es a pesar de publicar piezas con cierta regularidad no cuenta con equipos ni espacios específicos, sino que la producción se realiza desde el departamento de Infografía.

Civio y Data'n'Press fueron las plataformas productoras de proyectos de PD identificadas fuera de los medios. Ambas cuentan con equipos multidisciplinares y han elaborado piezas muy reconocidas, como 'Donde van mis impuestos' o 'España en Llamas' o 'Twittercatala.org'.

Data'n'Press ha colaborado con varios medios (El Confidencial.com, Elperiodico.es...) incluidos en la muestra en la elaboración de proyectos de PD; al tiempo que también hemos visto cómo medios como Elpais.com o Elconfidencial.com publican investigaciones basadas en grandes volúmenes de datos elaboradas por otras instituciones, como el Consorcio Internacional de Periodistas de Investigación (ICIJ). Una vía, la colaboración, que parece dar sus frutos; contribuye a la difusión de esta disciplina y cubriría la falta de recursos técnicos y personales que pudieran tener los medios en este ámbito.

Por otra parte, los profesionales encuestados estiman que falta de recursos económicos, de rentabilidad, y de formación de las redacciones, así como las propias estructuras de las redacciones, nada transversales, serían las razones que han lastrado el desarrollo de esta disciplina.

En todo caso, se aprecia un interés creciente, tanto en los medios como fuera, por las informaciones, visualizaciones o aplicaciones de noticias surgidas tras el análisis de enormes bases de datos. 


\section{Referencias bibliográficas}

BOUNEGRU, Liliana (2012): "Data Journalism in prespective", en GRAY, Jonathan; BOUNEGRU, Liliana; and CHAMBERS, Lucy (Eds.): The Data Journalism Handbook. Reino Unido, European Journalism Centre and the O'Reilly Media. http://interactivos.lanacion.com.ar/manual-data/introducci\%C3\%B3n_4.html [Consulta: 2 de julio de 2014]

BUENO, Ana (2014): "Periodismo de Datos: Antecedentes, ámbitos de desarrollo y aplicación en diarios generalistas: el caso de El Confidencial y El Periódico". Trabajo Fin de Grado. Universidad de Valladolid.

BRADSHAW, Paul (2012): "What is Data Journalism?", en GRAY, Jonathan; BOUNEGRU, Liliana; and CHAMBERS, Lucy (Eds.): The Data Journalism Handbook. Reino Unido, European Journalism Centre and the O'Reilly Media. http://interactivos.lanacion.com.ar/manual-data/introducci\%C3\%B3n_0.html [Consulta: 2 de julio de 2014]

BRADSHAW, Paul (2011): “The inveterted piramyd of data journalism" en Onlinejournalism.com, julio: http://onlinejournalismblog.com/2011/07/07/the-invertedpyramid-of-data-journalism/ [Consulta: 20 de julio de 2014]

CRUCIANELLI, Sandra (2012 a): "Introducción al Periodismo de Datos", en Internacional Center for Journalist: http://ijnet.org/es/blog/claves-para-entender-el-periodismo-de-datos [Consulta: 10 de julio de 2014]

CRUCIANELLI, Sandra (2012 b): "Periodismo de datos: lo bueno, lo malo y lo feo", en lanaciondata.com, junio: http://blogs.lanacion.com.ar/data/periodismo-conbases-de-datos/periodismo-de-datos-lo-malo-lo-bueno-y-lo-feo/ [Consulta: 14 de septiembre de 2014]

DADER, José Luis (1997): Periodismo de precisión. Vía socioinformática de descubrir noticias. Madrid, Síntesis.

FERRERAS, Eva María (2013): “Aproximación teórica al perfil profesional del «Periodista de datos», Icono 14, vol. 11 (2), pp. 115-140. Doi: 10.7195/ri14.v11i2.573

FLORES, Jesús y CEBRIÁN, Mariano (2012): "El data journalism en la construcción de mashups para medios digitales", en SABÉS, Fernando y VERÓN, José Juan (Coord.): El periodismo digital analizado desde la investigación procedente del ámbito académico. Huesca, XIII Congreso de Periodismo Digital, pp. 264-275.

FLORES, Jesús y SALINAS, Cecilia (2012): "Sinergias en la construcción del Nuevo Periodismo derivadas del Data Journalism y el Transmedia Journalism". Salamanca, III Congreso Internacional Comunicación 3.0. http://www.2ip.es/wpcontent/uploads/2013/01/sinergias_construccion_nuevoperiodismo.pdf [Consulta: 3 de septiembre 2014]

GORDON, Rich. (2007): "Data as journalism, journalism as data", en Readership Institute, noviembre: http://www.readership.org/blog2/2007/11/data-as-journalismjournalism-as-data.html [Consulta: 14 de julio de 2014] 
GRAY, Jonathan; BOUNEGRU, Liliana; and CHAMBERS, Lucy (Eds., 2012): The Data Journalism Handbook. Reino Unido, European Journalism Centre and the O'Reilly Media. http://datajournalismhandbook.org/ [Consulta: 4 de julio de 2014]

HOLOVATY, Andrew. (2006): “A Fundamental way newspaper sites need to change", en Holovaty.com, September: http://www.holovaty.com/writing/fundamentalchange/ [Consulta: 10 de junio de 2014]

ASOCIACIÓN DE LA PRENSA DE MADRID (2014): Informe Anual de la Profesión Periodística. Madrid.

MCGHEE, Geoff (2010): "Journalism in the Age of Data: A Video Report on Data Visualization", en Datajournalism.stanford.edu. http://datajournalism.stanford.edu/ [Consulta: 3 de julio 2014]

MEYER, Philip (1993): Periodismo de precisión: Nuevas fronteras para la investigación periodística. Barcelona, Bosch.

PEIRÓ, Karma y GUALLAR, Javier (2013): "Introducció al periodisme de dades. Característiques, panoràmica i exemples", Ítem, núm. 57, pp. 23-37. Barcelona, Colegi Oficial de Bibliotecaris-Documentalistes de Catalunya.

PERRY, Felipe y PAZ, Miguel (Eds, 2014): Manual de Periodismo de Datos Iberoamericano. Fundación Poderomedia. En: http://manual.periodismodedatos.org /index.php [Consulta: 3 de mayo 2014]

RODRÍGUEZ, Anidelys y GARCÍA Leidys (2014): "Cartografiando el periodismo de datos. Big Data y periodismo en el continente americano". Telos, núm. 95, pp. 57-67. Madrid, Fundación Telefónica.

ROGERS, Simon (2011): Facts are Sacred: The power of data. Guardian Shorts. Edición para Kindle.

ZANCHELLI, Michael y CRUCIANELLI, Sandra. (2012): Integrando el periodismo de datos en las salas de redacción. Washington, International Center for Journalism.

Eva María Ferreras Rodríguez es periodista (ha sido redactora en Europa Press y responsable de prensa en el Sindicato de Enfermería SATSE Euskadi). Es licenciada en Publicidad y Relaciones Públicas por la Universidad Complutense, licenciada en Periodismo por la Universidad Carlos III y Doctora en Periodismo por la Universidad del País Vasco. Ha sido profesora del Grado en Publicidad, Relaciones Públicas y Marketing del Centro Universitario CESINE (Santander, Cantabria). 